\title{
Real-time Compressing Algorithm based on Outer-trajectory Measurement Data
}

Jin Mei Wu ${ }^{a}$, Xiao Dong Ling, Shang Cheng Hu, Zhen Ping Wang

China Satellite Maritime Tracking and Controlling Department, Jiangyin 214413, Jiangsu, China

\begin{abstract}
Since huge sample datum has to be compressed properly in pre-processing to be sent out, a good compression algorithm will evidently improve the precision of the data-processing. In this paper, a compressing algorithm was studied based on polynomial fitting method. During the process of the real-time trajectory data compression, datasets were successively accumulated according to compression ratio. To apply all the information in the dataset, a series of orthogonal polynomial basis were applied to fitting the function, the least square estimation method was used to filter noise, and the estimated values of the position and the speed from differentiation of object datum in the dataset were sent out as compressed datum. And to get the best filter parameters, the mathematical expression of the error expectations and variances were studied. The compressing principle was given by considering the truncation error and random error simultaneously, which showed that, the best filter was the one by 21-point 3-order polynomial for position data compressing, while for speed data the filter by 41 -point 2-order polynomial was better. The theoretical analysis and the simulation results were also provided to prove the effectiveness of this algorithm in data-compression and noise filtering.
\end{abstract}

Keywords: Outer-trajectory measurement data ; Polynomial fitting; Compressing; Noise filtering

\section{Introduction}

Nowadays every kind of outer-measurement equipment collects data in some fixed sampling frequency. But in data processing, sampling frequency is often amplified. It is obligatory to compress data in preprocessing. Traditional method is giving up the rest points. Obviously this method wastes a large number of data and easily leads into new error. Frequently-used method averages all points, but is still unable to approach the real value. Whereas polynomial fitting is widely applied in time domain filtering and signal detection. In this paper, we study how the polynomial fitting can be presented into real-time trajectory data compression.

\section{Definition}

In real-time processing, a dataset accumulates and then the estimated value of position data in centre point on three coordinates and speed data from differentiation are outputting. Repeat to complete the compression from huge data to one point. In every dataset, a series of orthogonal polynomial basis were presented and the smoother formula of output point in every dataset with the least square filtering was applied to use every data fully and filter the measure noise.

${ }^{\mathrm{a} C}$ Corresponding author: wjm_83@aliyun.com 
Suppose sampling interval is $h$; sampling time is $t_{i}=i h$ in every dataset, $i=1, \mathrm{~L}, n$; flying goal motion function is $f(t)$, sampling equipment measurement value is $y_{i}$ at $t_{i}$, so $f\left(t_{i}\right)+\varepsilon_{i}$, in which $\varepsilon_{i}$ is random error. On the basis of Weierstrass Nol approximation theorem, $f(t)$ can be fitted by some m-order polynomial, such as

$$
y_{i}=\alpha_{0} P_{0}\left(t_{i}\right)+\alpha_{1} P_{1}\left(t_{i}\right)+\mathrm{L}+\alpha_{m} P_{m}\left(t_{i}\right)+\varepsilon_{i} \quad(1)
$$

Begin with uncomplicated algebra polynomial $\left\{1, t, t^{2}, \mathrm{~L}, t^{m}\right\}$, we construct orthogonal polynomial foundation. If $\mathrm{P}$ is the assemblage of all polynomial, any $p, q \in \mathrm{P}$, define the scalar product $\langle p, q\rangle=\sum_{i=1}^{n} p\left(t_{i}\right) q\left(t_{i}\right), \quad$ if $\langle p, q\rangle=0, p, q$ are orthogonal. By Schmidt orthogonalization method, we can get :

$$
\begin{aligned}
p_{0} & =1 \\
p_{1} & =t-\frac{n+1}{2} h \\
p_{2} & =t^{2}-(n+1) h t-\frac{n^{2}+3 n+2}{6} h^{2}=\left(t-\frac{n+1}{2} h\right)^{2}+\frac{1-n^{2}}{12} h^{2} \\
p_{3} & =t^{3}-\frac{3(n+1)}{2} h t^{2}-\frac{6 n^{2}+15 n+11}{10} h^{2} t-\frac{\left(n^{2}+5 n+6\right)(n+1)}{20} h^{3} \\
& =\left(t-\frac{n+1}{2} h\right)^{3}+\frac{7-3 n^{2}}{20} h^{2}\left(t-\frac{n+1}{2} h\right)
\end{aligned}
$$

If

$$
\begin{aligned}
& Y=\left(y_{1}, y_{2}, \mathrm{~L}, y_{n}\right)^{\tau}, \\
& X=\left(\begin{array}{ccc}
p_{0}\left(t_{1}\right) & \mathrm{L} & p_{m}\left(t_{1}\right) \\
\mathrm{M} & \mathrm{O} & \mathrm{M} \\
p_{0}\left(t_{n}\right) & \mathrm{L} & p_{m}\left(t_{n}\right)
\end{array}\right)_{n \times(m+1),} \\
& \alpha=\left(\alpha_{0}, \alpha_{1}, \mathrm{~L}, \alpha_{m}\right)^{\tau}, \text { consequently } Y=X \alpha \text {, and the }
\end{aligned}
$$

least-square estimate $\hat{\alpha}=\left(X^{\tau} X\right)^{-1} X^{\tau} Y$,where $X^{\tau} X=\operatorname{diag}\left(S_{0}, S_{1}, \mathrm{~L}, S_{m}\right), S_{j}=\left\langle p_{j}, p_{j}\right\rangle$, get that:

$S_{0}=n$

$S_{1}=\frac{n\left(n^{2}-1\right) h^{2}}{12}$

$S_{2}=\frac{n\left(n^{2}-1\right)\left(n^{2}-4\right) h^{4}}{180}$

$S_{3}=\frac{n\left(n^{2}-1\right)\left(n^{2}-4\right)\left(n^{2}-9\right) h^{6}}{2800}$

So we get that

$$
\hat{\alpha}=\left(\sum_{i=1}^{n} p_{0}\left(t_{i}\right) y_{i} S_{0}^{-1}, \sum_{i=1}^{n} p_{1}\left(t_{i}\right) y_{i} S_{1}^{-1}, \mathrm{~L}, \sum_{i=1}^{n} p_{m}\left(t_{i}\right) y_{i} S_{m}^{-1}\right)
$$

Smoother formula of position data and speed data at output point are:

$\hat{f}(t)=\sum_{j=0}^{m} \hat{\alpha}_{j} p_{j}(t)=\sum_{j=0}^{m}\left(\sum_{i=1}^{n} p_{j}\left(t_{i}\right) y_{i} S_{j}^{-1}\right) p_{j}(t)=\sum_{i=1}^{n}\left(\sum_{j=0}^{m} p_{j}\left(t_{i}\right) S_{j}^{-1} p_{j}(t)\right) y_{i}{ }^{(5)}$

$\hat{f}^{\prime}(t)=\sum_{j=0}^{m}\left(\sum_{i=1}^{n} p_{j}\left(t_{i}\right) y_{i} S_{j}^{-1}\right) p_{j}^{\prime}(t)=\sum_{i=1}^{n}\left(\sum_{j=0}^{m} p_{j}\left(t_{i}\right) S_{j}^{-1} p_{j}^{\prime}(t)\right) y_{i}{ }^{(6)}$

\section{Example}

We choose theoretical value and mixed noise to produced outer-measurement data. In Matlab simulation environment, we choose component product in $\mathrm{X}$ axis coordinate, then mixed a set of white noise with 0 mean value 10 variance, and select data of 250 seconds. Compare with four methods: choosing second points, averaging, 3order 21 point polynomial fitting, 3order 41 point polynomial fitting. Results showed as pictures. 


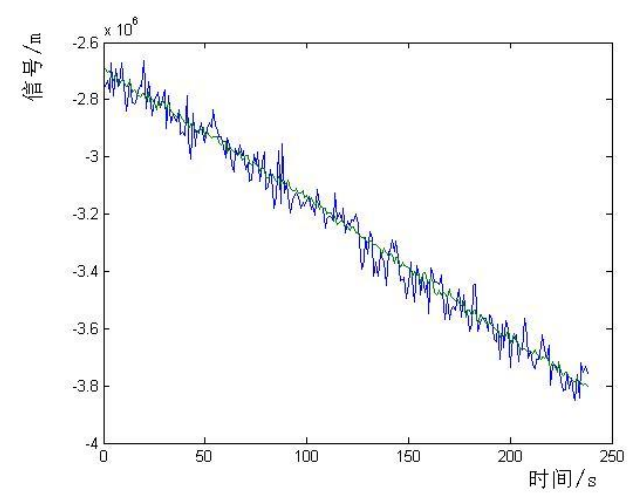

Fig. 1 Comparison of simulation data and compressed signals fitted by 21-point 3-order polynomial

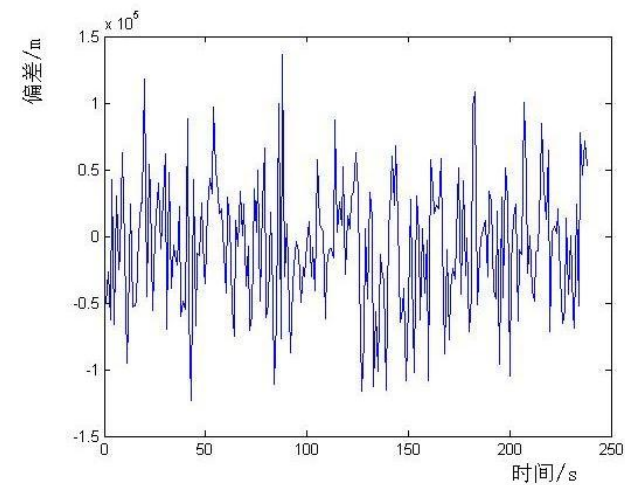

Fig. 2 Error of output data every second

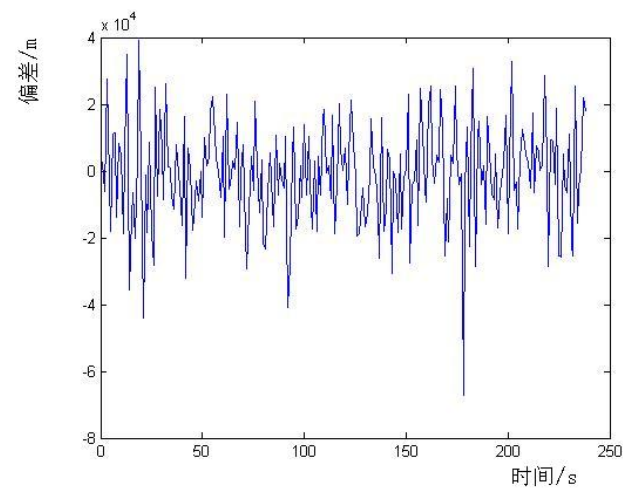

Fig. 4 Error of data fitted by 41-point 3-order polynomial

We can perceive that 3 order21point polynomial fitting compression method reflect data characteristic, the error with real value obviously is smaller than methods of choosing second points and averaging.

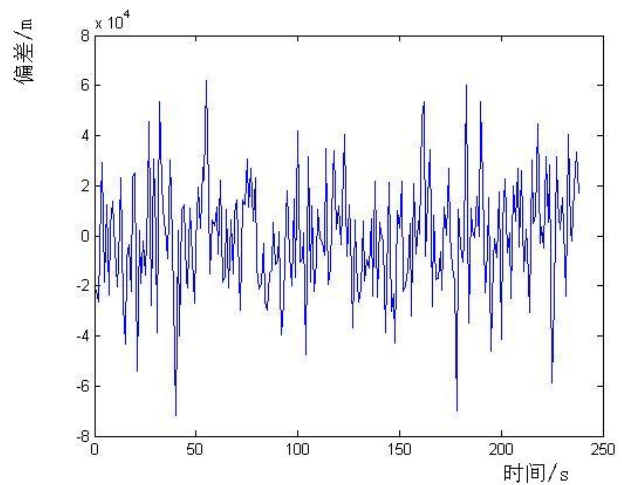

Fig. 3 Error of average method

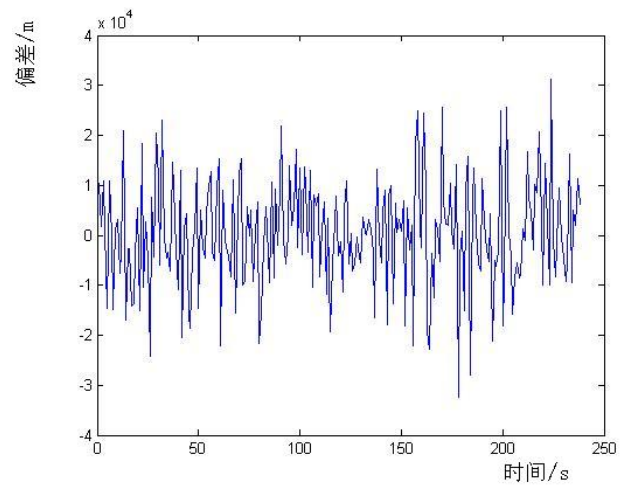

Fig. 5 Error of data fitted by 21-point 3-order polynomial

\section{Conclusion}

Using polynomial fitting to compress outer-measurement data is uncomplicated, practical and can satisfy the requirement of real-time 
outer-measurement preprocessing. As the simulation result shows, the novel algorithm is a promised compression and noise filtering method which can reflect real curve characteristic in higher precision.

\section{References}

[1] Zhenjun An, Min Wang, Shaolin HU, et al, Tracking Data Collection and Processing Technologies, Journal of Spacecraft TT\&C Technology, 2005,24(2):56-60.

[2] Lisheng Liu, Data Processing of Outer-Trajectory Measurement, Beijing:National Defense Industry Press,2002:151-170.

[3] Zhengming Wang, Haiying Zhou. Several Calculating Problems of the Processing of Trajectory Tracking Data, Journal of National University of Defense Technology, 2001, 23(6):42-47.

[4] Bo Yu, Wei Jian, Jianxun Chen, et al, A Method Based on Orthogonal Polynomial Partitioned Imitation for Image Compression Coding , Microcomputer Application, 2007,28(12):1316-1320.

[5] Zuoping Qin, Puyin Liu. Non-central Differentially Moving Average With Polynomial, Journal of Astronautics, 1997, 18(2):43-49. 